\title{
NOTE TECHNIQUE \\ vitesse de déformation et essais quasi statiques
}

\author{
M. SAHLI \\ Docteur ès Sciences Techniques, \\ Ecole Hassania des Travaux Publics
}

Rev. Franç. Géotech. n 51, pp. 69-73 (avril 1990)

Les essais quasi statiques à vitesse de déformation constante sont des essais de laboratoire courants. En mécanique des roches notamment, le comportement du matériau testé dépend de la vitesse de déformation imposée par la presse [SAHLl, 1988]. Pour certains auteurs cette dépendance peut être en relation avec le comportement visqueux des géomatériaux. Cependant, l'étude précise des mécanismes de déformation au sein du géomatériau, entre autres, amène à penser que cette hypothèse n'est pas plausible.

\section{MÉCANISMES DE DÉFORMATION DES ROCHES COMPACTES}

La déformation des roches fait appel à un processus de mécanismes complexes, difficiles à mettre en évidence. La connaissance de tels mécanismes est pourtant essentielle à une bonne compréhension du comportement des roches et à une formulation théorique de celui-ci.

A l'instar des métaux, les microstructures matricielles, dislocations, micropores, microfissures et leurs orientations préférentielles déterminent la résistance et la rupture des roches [SAHLI, 1988].

Il y a ainsi un rapport étroit entre l'échelle microscopique et les propriétés mécaniques des roches compactes [ALM, 1982 ; BERGUES et al., 1984 ; SUAREZ DEL RIO, 1985 ; WENK, 1982]. Quand un échantillon est sollicité jusqu'à sa ruine, les mécanismes de déformation en mode local sont attribués à la coalescence de la microfissuration induite en cours de déformation, pour former des zones de glissement potentielles au sein de la matrice rocheuse sous charge [DAFALIAS, 1985 ; DESRUES, 1987 ; MEHRABADI et COWIN, 1980 ; MROZ, 1985 ; PISTER, 1985 ; TEUFEL, 1985].

Dans le cas des essais différés, les mécanismes sont moins bien connus. Il est possible qu'en fluage transitoire il y ait compétition entre les déformations par dislocations et par microfissuration [CRUDEN, 1971 ; HOFER et KNOLL, 1971]. Alors que le fluage permanent ne peut être dú qu'à des mouvements de dislocations, le fluage tertiaire a lieu par amplification de la microfissuration et formation de zones de glisssement au sein de la matrice.

\section{ROCHE TESTÉE}

Il s'agit d'une molasse gréseuse constituant le Plateau suisse entre le Jura et les Préalpes. Cette roche fait l'objet de travaux expérimentaux importants à cause de sa fréquence et de sa présence dans les travaux souterrains exécutés dans la région.

D'aspect homogène, non fissuré et de consistance friable, le bloc à l'origine des échantillons de laboratoire a été extrait dans la carrière de Villarlod et de Massonens dans le canton de Fribourg.

Au laboratoire, des échantillons de diamètre $55 \mathrm{~mm}$ et d'élancement 2 ont été taillés et préparés dans des conditions semblables. On ne retrouve évidemment pas l'état de la roche in situ, mais le contrôle et le stockage des échantillons a permis de limiter la plage de température et de teneur en eau dans des marges étroites.

Tableau 1. - Identification de la roche étudiée.

\begin{tabular}{|c|c|}
\hline $\begin{array}{l}\text { Poids volumique sec } \\
\text { Poids spécifique } \\
\text { Teneur en eau de saturation } \\
\text { Porosité équivalente } \\
\text { Perméabilité à l'eau } \\
\text { Composition minéralogique } \\
\begin{array}{lr}\text { - quartz } & 67 \% \\
\text { - carbonates } & 28 \% \\
\text { - argiles } & 5 \%\end{array}\end{array}$ & $\begin{array}{c}22 \mathrm{kN} / \mathrm{m}^{3} \\
26,5 \mathrm{kN} / \mathrm{m}^{3} \\
6 \% \text { environ } \\
17 \% \\
1,2.10^{-9} \mathrm{~m} / \mathrm{s}\end{array}$ \\
\hline
\end{tabular}

Tableau 2. - Influence de l'humidité relative de stockage sur la teneur en eau de la roche testée.

\begin{tabular}{|l|l|l|l|l|l|l|}
\hline $\begin{array}{l}\text { Humidité } \\
\text { relative (\%) }\end{array}$ & 9 & 33 & 55 & 81 & 93 & 100 \\
\hline $\begin{array}{l}\text { Teneur } \\
\text { en eau (\%) }\end{array}$ & 0,2 & 0,5 & 0,6 & 0,8 & 0,9 & 5,8 \\
\hline
\end{tabular}


En conclusion, la roche étudiée est sensible aux conditions hydriques de stockage. Cependant, en cours d'essais, la teneur en eau a pu être gardée dans une fourchette étroite : 0,6 à $1 \%$, correspondant à une variation de l'humidité relative entre 55 et $95 \%$. Ceci explique la faible dispersion obtenue dans les essais effectués au laboratoire sur cette roche.

\section{RÉSULTATS DES ESSAIS} DE CHARGE EFFECTUÉS [SAHLI, 1988]

Les déformations sont mesurées entre plateaux par comparateur magnétique. La presse utilisée est de type rigide d'une capacité de $500 \mathrm{kN}$. Le vérin est asservi en déformation. L'acquisition, l'interprétation des données et les représentations graphiques sont effectuées sur ordinateur. On a choisi de ne pas rapporter l'ensemble des courbes obtenues au cours de ce travail. Dans un but didactique, on donne en figure 1 un exemple de courbe $\sigma-\epsilon$ obtenue, ainsi que le module tangent calculé par ajustement linéaire entre 20 et $80 \%$ de la valeur $R_{c}$ de l'échantillon.

Les résultats des essais effectués sont présentés dans le tableau 3 ci-après

Les calculs statistiques sont résumés dans le tableau 4 ci-après.

L'ajustement linéaire des divers points expérimentaux est donné en figure 2 .

L'ajustement linéaire des points expérimentaux fait ressortir une corrélation semi-logarithmique, dont la loi est approximativement :

$$
R_{c}=0,36 \log _{10}(\epsilon)+13,7
$$

$-\stackrel{\bullet}{\epsilon}$ en $\mathrm{s}^{-1}$

- $\mathrm{R}_{\mathrm{c}}$ en $\mathrm{MPa}$

Les modules $\mathrm{E}_{\mathrm{c}}$ ne semblent pas varier avec $\stackrel{0}{\text {. }}$

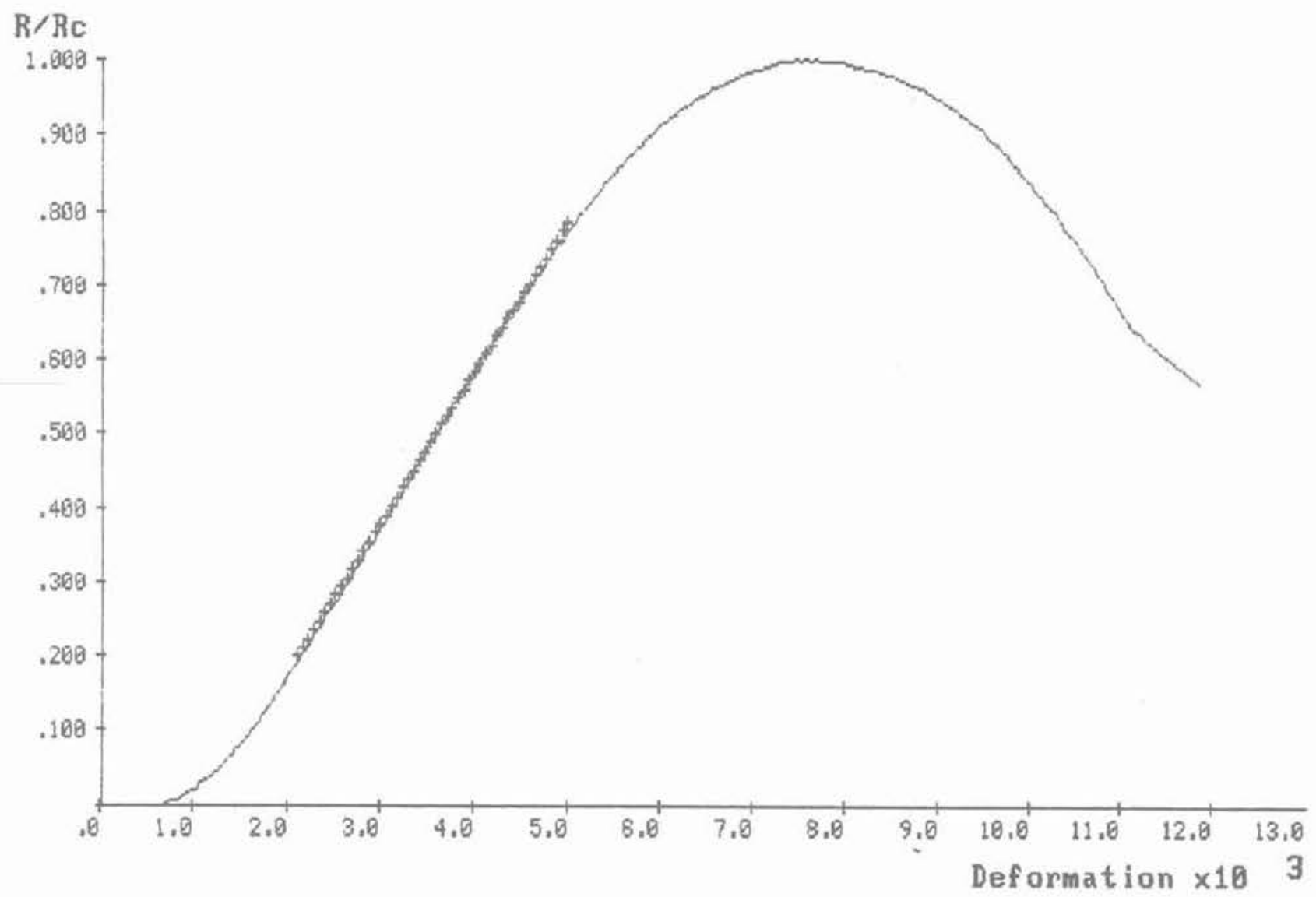

Fig. 1. - Exemple de courbe $\sigma-\epsilon$ en déformation monotone.

Echantillon $\times 0331: \dot{\epsilon}=410^{-5} s^{-1} \cdot E c=2540 \mathrm{MPa}-R_{c}=12,5 \mathrm{MPa}$ 
Tableau 3. - Résistance et module de chargement en fonction de $\epsilon_{\text {. }}$

\begin{tabular}{|c|c|c|}
\hline$\dot{\epsilon}\left[s^{-1}\right]$ & $R_{\mathrm{c}}[\mathrm{MPa}]$ & $E_{\mathrm{c}}[\mathrm{MPa}]$ \\
\hline $0,3210^{-6}$ & 11,3 & 2200 \\
$0,32 "$ & 11,6 & 2203 \\
$0,32 "$ & 11,3 & 2130 \\
$0,32 "$ & 11,6 & 2487 \\
$0,32 "$ & 10,8 & 2277 \\
$0,32 "$ & 11,4 & 2201 \\
$0,3910^{-4}$ & 12,1 & 2322 \\
$0,39 "$ & 11,7 & 1957 \\
$0,39 "$ & 12,6 & 2414 \\
$0,39 "$ & 11,6 & 2365 \\
$0,39 "$ & 11,9 & 2507 \\
$0,39 "$ & 12,5 & 2531 \\
$0,4410^{-3}$ & 13,1 & 2049 \\
$0,40 "$ & 13 & 2203 \\
$0,42 "$ & 12,7 & 1987 \\
$0,32 "$ & 11,6 & 2441 \\
$0,28 "$ & 13 & 2168 \\
$0,22 "$ & 12,6 & 2317 \\
$0,43 "$ & 11,9 & 2097 \\
$0,34 "$ & 12,4 & 2254 \\
$0,1710^{-2}$ & 12,7 & 2341 \\
$0,15 "$ & 12,7 & 2182 \\
$0,14 "$ & 13,3 & 2515 \\
$0,36 "$ & 12,4 & 2316 \\
$0,34 "$ & 13 & 2460 \\
$0,35 "$ & 12,4 & 2116 \\
$0,37 "$ & 13,4 & 2156 \\
$0,34 "$ & 12,7 & 2068 \\
$0,43 "$ & 12 & \\
\hline
\end{tabular}

Tableau 4. - Calculs statistiques sur les données du tableau 3.

\begin{tabular}{|c|c|c|c|c|c|}
\hline \multirow{2}{*}{$\epsilon^{0}\left[s^{-1}\right]$} & \multirow{2}{*}{$\begin{array}{c}\text { Nombre } \\
\text { d'essais }\end{array}$} & \multicolumn{2}{|c|}{$\mathrm{R}_{\mathrm{c}}[\mathrm{MPa}]$} & \multicolumn{2}{c|}{$E_{\mathrm{c}}[\mathrm{MPa}]$} \\
\cline { 3 - 6 } & & moyenne & $\mathrm{CV}[\%]$ & moyenne & $\mathrm{CV}[\%]$ \\
\hline $3,610^{-3}$ & 7 & 12,7 & 4 & 2270 & 8 \\
$1,410^{-3}$ & 4 & 13 & 2 & 2350 & 7 \\
$0,410^{-3}$ & 8 & 12,7 & 3 & 2150 & 5 \\
$0,410^{-4}$ & 6 & 12,1 & 3 & 2350 & 9 \\
$0,310^{-6}$ & 7 & 11 & 3 & 2280 & 6 \\
\hline
\end{tabular}

CV : coefficent de variation de la variable aléatoire.

\section{INTERPRÉTATION}

La vitesse de déformation joue un rôle sur la résistance à la rupture des échantillons, alors que les modules de charge semblent inchangés. Cette dernière invariance est confirmée par le faible coefficient de variation associé à l'ensemble des modules mesurés, de $7,3 \%$.

Que les modules de charge tangents ne soient pas fonction de la vitesse de charge semble avoir échappé aux expérimenteurs en mécanique des roches jusquelà.

L'effet de la vitesse de charge sur le pic de la rupture en compression simple, observé ici, a été confirmé par ailleurs [SAHLI, 1988]. Cependant, contrai-

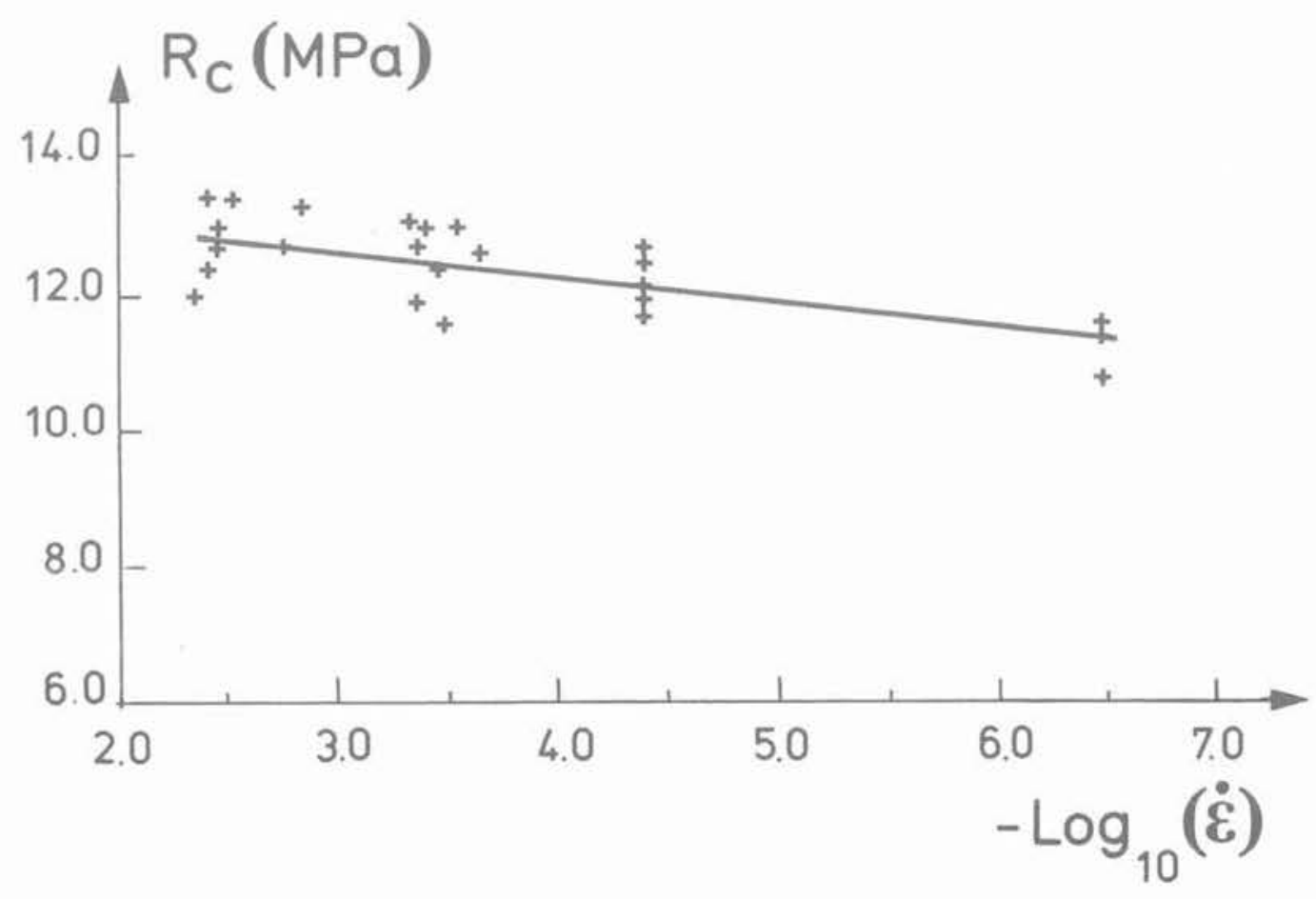

Fig. 2. - Variation de $R_{c}$ avec la vitesse de déformation $\AA_{\text {. }}$ 


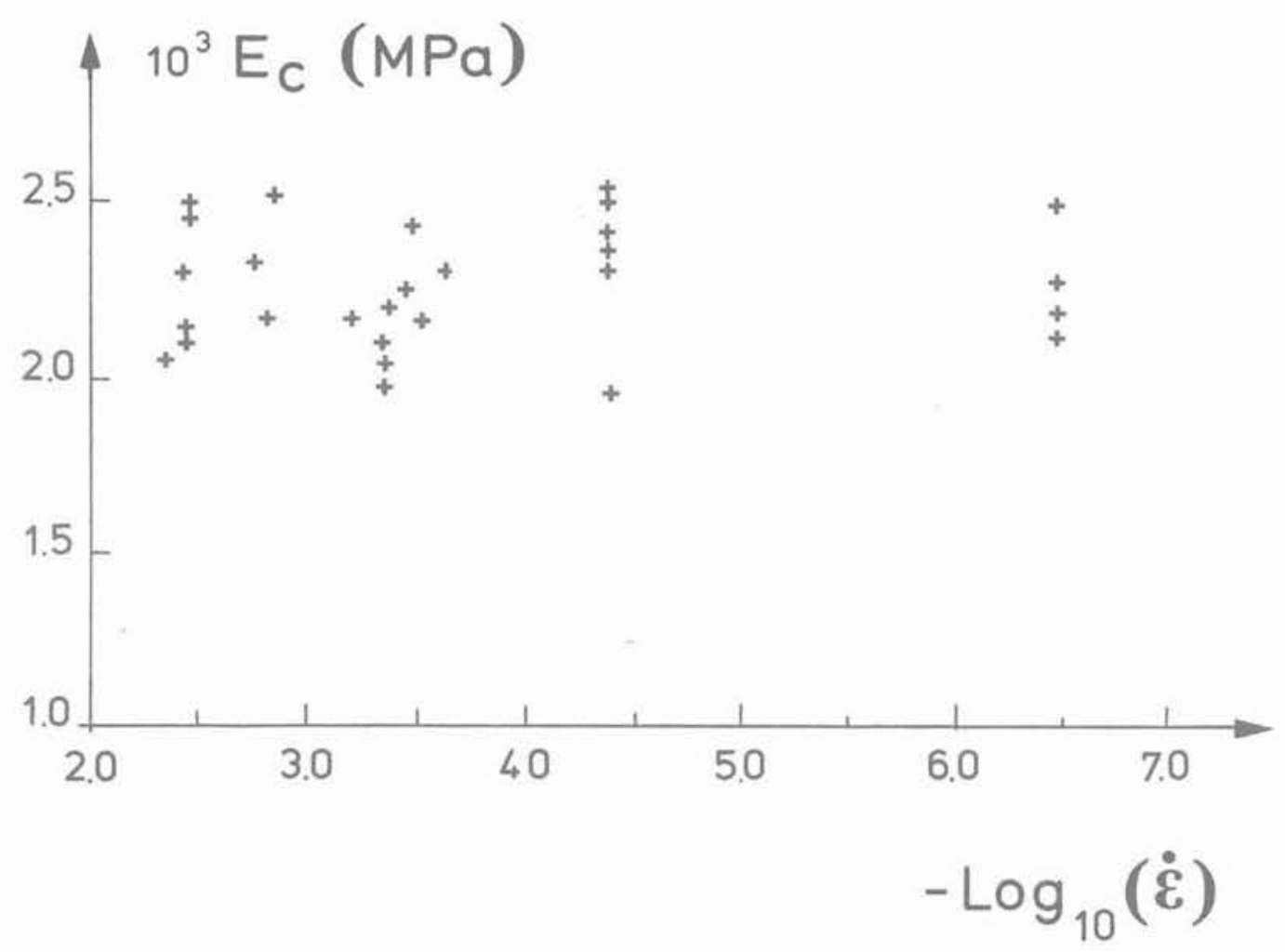

Fig. 3. $-E_{c}$ en fonction de la vitesse de déformation $\stackrel{e}{e}$

rement à ce qui a été avancé parfois, le comportement visqueux n'est pas nécessairement l'explication de cette dépendance. Nous pensons que la relation entre les contraintes de pic en compression simple et la vitesse de déformation est due aux mécanismes de déformation en jeu et au développement de la microfissuration dont la vitesse est en compétition avec la vitesse d'écrasement. Cette hypothèse est suggérée par l'invariance du module de charge, les mécanismes de déformation en jeu au voisinage du pic, ainsi que par des études de comportement différé de la roche étudiée [SAHLI, 1988].

\section{REMERCIEMENTS}

Cette étude a été réalisée dans le cadre d'un travail de recherche au Laboratoire de Mécanique des Roches de l'Ecole Polytechnique Fédérale de Lausanne (Suisse), sous la direction du Professeur F. DESCOEUDRES. A ce dernier et à son équipe vont mes remerciements et ma reconnaissance.

\section{BIBLIOGRAPHIE}

ALM O. (1982), The effect of water on the mechanical properties and microstructures of granitic rocks at high pressures and high temperatures. Issues in Rock Mechanics, 23rd symposium on rock mechanics, Berkeley.
BERGUES J., CHARPENTIER J.P., HABIB P. (1984), Influence de la fissuration sur le comportement mécanique du granite et effets de la tem. pérature. Journées sur le granite, Orléans-la Source, 26 juin, pp. 203-212, Document BRGM.

CRUDEN D.M. (1971), Single-increment creep experiments on rock under uniaxial compression. Int. J. Rock Mech. Min. Sci., Vol. 8, pp. 127-142, Pergamon Press.

DAFALIAS A.Y.F. (1985), A. Summary of discus. sions on constitutive modelling of nonlinear triaxial behaviour (part II). Mechanics of Geomaterials, Rocks, Concrete, Soils edited by Z.P. BAZANT, pp. 569-570, John WILEY and Sons.

DESRUES J. (1987). Naissance des bandes de cisaillement dans les milieux granulaires: expérience et théorie. Manuel de rhéologie des géomatériaux, sous la direction de F. DARVE, Presses de I'ENPC, Paris.

HOFER K.H., KNOLL P. (1971), Investigations into the mechanism of creep deformation in cornallitite, and practical applications. Int. J. Rock Mech. Min. Sci., Vol. 8, pp. 61-73, Pergamon Press, 1971.

MEHRABADI M.M., COWIN S.C. (1980), Prefailure and postfailure soil plasticity models. Journal of the Engineering Mechanics Division, October 1980.

MICHELIS P. (1987), True triaxial yielding and hardening of rock. J. of Geotech. Engin., Vol. 113, $N^{\circ} 6$, Juin 1987, pp. 617-635. 
MROZ Z. (1985), Current problems and new directions in mechanics of geomaterials. Mechanics of Geomaterials, Rocks, Concrete, Soils edited by Z.P. BAZANT, pp. 539-566, John WILEY and Sons.

PISTER K.S. (1985), Some remarks on constitutive equations for concrete and geomaterials : discusser's report. Mechanics of Geomaterials, Rocks, Concrete, Soils edited by Z.P. BAZANT, pp. 461-468, John WILEY and Sons.

SAHLI M. (1988), Lois d'écoulement visqueux des géomatériaux-applications à un grès. Thèse de doctorat ès Sciences Techniques, Laboratoire de Mécanique des Roches, EPF Lausanne, Suisse.
SUAREZ DEL RIO L.M., NORIN J. (1985), Bending tests and acoustic emissions for heated Avesta. gneiss - technical note. Int. J. Rock Mech. Min. Sci. and Geomech. Abstr., vol. 22.

TEUFEL L.W. (1985), C. Summary of discussions on shear localization faulting, and frictional slip (part IV). Mechanics of Geomaterials, Rocks, Concrete, Soils edited by Z.P. BAZANT, pp. 575-756, John WILEY and Sons.

WENL H.R. (1982), Workshop on deformation mechanisms and texture development in rocks. Issues in Rock Mechanics, 23rd symposium on rock mechanics, Berkeley. 